\title{
Atomic and Electronic Interface Structure of a VN Hard Coating on MgO
}

\author{
Zaoli Zhang $^{1}$, B. Raskova ${ }^{1}$, G. Dehm ${ }^{1,2}$, P. Lazar ${ }^{3}$, J. Redinger $^{3}$, R. Podloucky ${ }^{4}$
}

1. Erich Schmid Institute of Materials Science, Austrian Academy of Sciences, Leoben,

2. Department Materials Physics, University of Leoben, Leoben, Austria

3. Institute of Applied Physics, Vienna University of Technology, Vienna, Austria

4. Department of Physical Chemistry, Vienna University, Vienna, Austria

Hard coatings are important for triboligical applications where wear and friction are important. One of the aims in coating design is the development of super-hard coatings. This can be achieved if interfaces are tailored with respect to their bond strength. In this study we report a quantitative comparison of the interface structure of a VN coating grown epitaxially on a $\mathrm{MgO}(001)$ substrate using aberration-corrected high resolution transmission electron microscopy (HRTEM), electron energy loss spectroscopy (EELS), and ab-initio density functional theory (DFT). By Cs-corrected HRTEM, we clearly resolved the oxygen and nitrogen sublattices across the VN/MgO interface. $\mathrm{As}$ revealed by DFT, the (002) interplanar spacing oscillates in the first several VN layers across the interface. The insights gained by advanced TEM firmly corroborate that the interfacial structure within a few atomic layers slightly deviates from both bulk sides. Interfacial chemistry determined by EELS analysis shows the preponderance of $\mathrm{O}$ and $\mathrm{V}$ atom at the interface, resulting in a small detectable core-level shift. It can be speculated that these structure oscillations may be important for tuning the elastic properties of interface controlled materials such as nanostructured hard coatings.

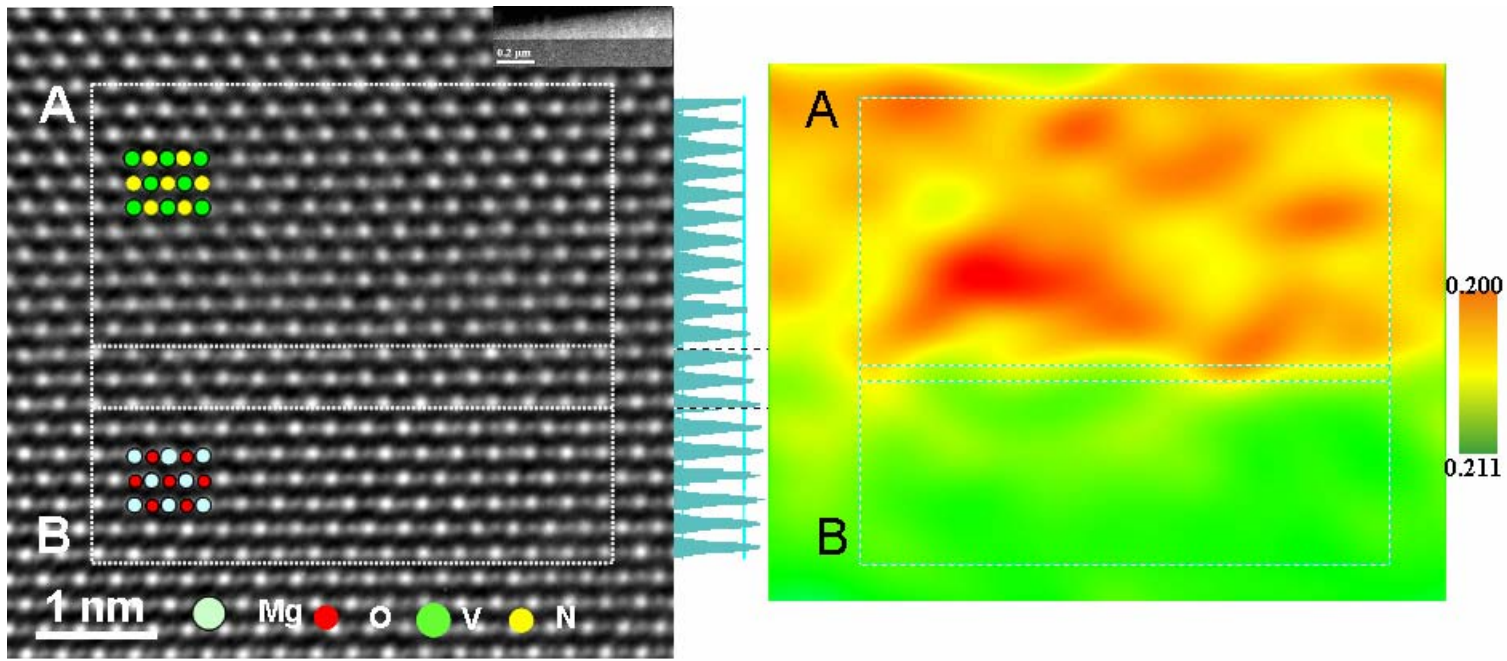

(a) HRTEM image of the VN/MgO interface with an epitaxial relationship, i.e. $<100>(001)$ $\mathrm{VN} / /<100>(001) \mathrm{MgO}$, recorded along [110] direction using a negative $\mathrm{C}_{\mathrm{S}}(-3.0 \mu \mathrm{m})$, in which the $\mathrm{O}$ and $\mathrm{N}$ atomic columns are clearly visible. The intensity profile (from $\mathrm{A}$ to $\mathrm{B}$ ) obtained by integrating over a rectangular area reveals a jump in the intensity across the interface. (b) Color map of modulation of the 002 spatial frequency, showing the $d_{002}$ spacing distribution across the interface. A low magnification ADF STEM image is inserted in (a). The colour code ranges from $0.200 \mathrm{~nm}$ to $0.211 \mathrm{~nm}$. 